IAC-06-B6.2.2

\title{
CALCULATING STATISTICAL ORBIT DISTRIBUTIONS USING GEO OPTICAL OBSERVATIONS WITH THE MICHIGAN ORBITAL DEBRIS SURVEY TELESCOPE (MODEST)
}

\author{
M. Matney \\ NASA Johnson Space Center, Houston, TX, USA \\ mark.matney-1@nasa.gov \\ K.J. Abercromby, H.M. Rodriguez \\ ESCG/Jacobs, Houston, TX, USA \\ kira.abercromby-1@nasa.gov, heather.m.rodriguez@nasa.gov \\ E. Barker \\ NASA Johnson Space Center, Houston, TX, USA \\ edwin.s.barker@nasa.gov \\ P. Seitzer \\ University of Michigan, Ann Arbor, MI, USA \\ pseitzer@umich.edu
}

\begin{abstract}
NASA's Orbital Debris measurements program has a goal to characterize the small debris environment in the geosynchronous orbit (GEO) region using optical telescopes ("small" refers to objects too small to catalog and track with current operational systems). Traditionally, observations of GEO and near-GEO objects involve following the object with the telescope long enough to obtain an orbit suitable for tracking purposes. Telescopes operating in survey mode, however, randomly observe objects that pass through their field-of-view. Typically, these short-arc observation are inadequate to obtain detailed orbits, but can be used to estimate approximate circular orbit elements (semi-major axis, inclination, and ascending node). From this information, it should be possible to make statistical inferences about the orbital distributions of the GEO population bright enough to be observed by the system. The Michigan Orbital Debris Survey Telescope (MODEST) has been making such statistical surveys of the GEO region for five years. During that time, the telescope has made sufficient observations in enough areas of the GEO belt to have achieved nearly complete coverage. That means that almost all objects in all possible orbits in the GEO and near-GEO region had a non-zero chance of being observed. Some regions (such as those near zero inclination) have had good coverage, while others are poorly covered. Nevertheless, it is possible to remove these statistical biases and reconstruct the orbit populations within the limits of sampling error. In this paper, these statistical techniques and assumptions are described, and the techniques are applied to the current MODEST data set to arrive at our best estimate of the GEO orbit population distribution.
\end{abstract}

\section{INTRODUCTION}

In recent years, there has been an increasing interest in the economically-important geosynchronous region of Earth orbit (GEO). For the purposes of this paper, "GEO" will not only refer to objects that maintain periods very close to the rotation period of the Earth, but to all objects in the GEO and near-GEO regime - all of which potentially could affect true GEO orbits by collisions or production of debris. This includes discarded rocket bodies with orbits below the true geosynchronous altitude and retired spacecraft that were boosted hundreds of kilometers above the orbits of operational GEO satellites.

Orbit lifetimes of objects left in the GEO region can exceed thousands of years, so if current 
launch and disposal behavior persists, discarded rocket bodies and nonfunctional spacecraft will continue to accumulate in the region for the foreseeable future. One of the primary sources of debris in low-Earth orbit (LEO) is from breakups of satellites (e.g., rocket bodies with unspent fuel can explode spontaneously). By analogy, similar explosions at GEO could be primary contributors to debris as well. We know that there have been at least two breakups in GEO, where the explosion of an object has created at least some debris ${ }^{1}$.

The United States Space Surveillance Network (SSN) maintains a catalog of objects in GEO that are regularly tracked by radar and optical instruments. Most of these objects, however, are intact spacecraft and rocket bodies. Breakup fragments are typically smaller than intact objects. At GEO ranges, such fragments are generally too small for the SSN to detect and track efficiently in order to maintain orbital elements. Therefore, very little is known about the population of fragmentation debris in the GEO regime.

In order to better characterize this fragment population, a number of nations are now using (or are developing) optical telescope systems to try to characterize the debris environment in $\mathrm{GEO}^{2,3,4}$. NASA's strategy has been to use optical telescopes in a survey mode to statistically sample the GEO environment. This is analogous to NASA's strategy for LEO, where highly sensitive radars are used to stare at a particular region in space and count small objects as they pass through the beam ${ }^{5}$.

This paper outlines the statistical methods used to transform individual detections of objects in the GEO regime into estimates of the actual orbit populations in space. We use the most recent data from the Michigan Orbital Debris Survey Telescope (MODEST) to determine the first good estimates of fragmentation debris in the GEO region.

\section{MEASUREMENTS}

The first systematic statistical observation of the GEO region was done using NASA's CCD Debris Telescope $(\mathrm{CDT})^{6}$. This $32 \mathrm{~cm}$ aperture
Schmidt telescope had a $1.7^{\circ}$ x $1.7^{\circ}$ field-ofview (FOV), and operated for several years, beginning in 1998. This was also the first data used to statistical orbit distributions of the GEO environment ${ }^{7}$.

The analysis of the CDT data highlighted several important data issues:

First, because the telescope stares at a particular patch of sky for an extended period of time, only certain orbits can be observed. In order to have a non-zero chance to see all possible orbits of interest, observations must be made over a number of different locations in the sky. The actual detection probability of an orbit is a function of the pointing direction of the telescope and the time it was used to observe. This introduces biases in the counting statistics, because some orbits are more likely to be seen than others. Therefore, if we are trying to extract the original populations, it is vital that these biases be understood and removed.

Second, the telescope makes only angles-only observations (right ascension and declination) of the objects it detects, and these measurements are used to compute and orbit. The measurements are typically too short to easily determine the true orbit of the object, so special techniques must be used to obtain useful results.

Due to limitations of the CDT telescope, it was only able to produce relatively-complete orbit populations down to about $15^{\text {th }}$ absolute magnitude (the visual magnitude of the object corrected to $36,000 \mathrm{~km}$ distance and $0^{\circ}$ solar phase angle).

In 2002, NASA began observations using the MODEST telescope ${ }^{9}$, a 0.6/0.9 m Curtis Schmidt telescope operated by the University of Michigan located at CTIO in Chile. It has a $1.3^{\circ} \mathrm{x} 1.3^{\circ}$ FOV, uses a 2048 x 2048 pixel thinned SITe CCD (Peak QE 90\%). Each pixel covering 2.3 arc seconds and the system can detect objects down to about $17^{\text {th }}$ or $18^{\text {th }}$ magnitude in brightness.

Observations are made at a single right ascension and declination over an observing period. Each frame is 4.9 seconds long and a 
new frame is taken every 37.9 seconds (the delay is due to readout time). The telescope tracks the fixed stars at the sidereal rate, but the CCD is read out in Time Delay Integration (TDI) mode (drift scanning) to "put back in" the Earth rotation. Consequently, in each frame the stars are streaked, but the same stars are visible in every frame. Using this method, GEO objects appear as points or short streaks across the image, so that the maximum light from the object is focused onto the fewest number of pixels. A "detection" is defined as the same object seen in 4 or more frames. Under the current observation mode, objects with motion rates exceeding \pm 2 " per second in Hour Angle or \pm 5 " per second in Declination are rejected (although this "rate box" can easily be modified). This limitation minimizes the possible number of high-eccentricity GEOtransfer orbit objects (GTOs) that can sometimes be mistaken for GEO objects over short arcs.

Observations are targeted near to the Earth shadow at GEO, so that the target objects will have minimum solar phase angle, but still be clear of the shadow. This is done to (hopefully) maximize the brightness of the objects. In addition, the observations are usually chosen to avoid the plane of the Milky Way as much as possible, for the multitude of stars in that region of the sky can easily mask dim GEO objects or overwhelm the automated object detection algorithm. Early in the program, the observations were made to maximize coverage of the catalogued GEO population, but over time we have tried to extend the observations to observe as many orbits as possible.

The data set used in this paper consists of approximately 130 nights, spanning the period of 2002-2006. It was necessary to include such a long time span in order to get relatively complete coverage of the GEO regime. The problem with using such data over such a long time is that the orbits of GEO objects precess slowly, taking about 50 years to complete one cycle. Consequently, the orbit planes of objects can move several degrees over that period of time. Ideally, complete surveys should be done over a time scale of about 1 year. However, this requires careful scheduling of telescope pointing and "cooperative" weather conditions.

Because this orbit precession was occurring during the 5 years of the MODEST observation program and the coverage in time and space was not uniform, there will be some imprecision in the computed orbit distributions. What we can assume is that the estimated populations represent a sort of time-averaged value of the population in a particular orbit over the period of observations. This will result in some blurring of the orbit distributions.

\section{MODELING}

The computation of orbits using arcs of anglesonly observations is one of the oldest problems in mathematical astronomy ${ }^{8}$. Because MODEST observations make observations over short arcs, it is sometimes difficult to determine whether the orbit is circular or elliptical. Currently, NASA is pursuing some encouraging methods to investigate whether the short-arc MODEST observations are sufficiently accurate to (at least) distinguish between nearly-circular orbits and highly elliptical GTO orbits. For this study, however, we use the Assumed Circular Orbit (ACO) fit for analysis.

Historically, orbits are defined by sixdimensional Kepler elements (there are several ways to represent these elements - all equivalent - but a typical set consists of the semi-major axis, eccentricity, inclination, ascending node, argument of perigee, and true anomaly, all at a given epoch). However, "moving around" in this six-dimensional space to optimize a data fit presents a number of difficulties. Kepler elements can have singularities in their derivatives, and even specialized "non-singular" elements have potential problems. There is a way of representing a state vector of an orbit that is non-singular, however, and that is by using Cartesian coordinates. There is a one-to-one correspondence between a six-dimensional Cartesian state vector ( 3 dimensions of position, 3 of velocity) and a set of Kepler elements. The six-dimensional Cartesian state vectors have continuous derivatives, unlike Keplerian elements, so it is easier to optimize the solution 
in "Cartesian space" and transform back into "Keplerian Space" after the fit is completed.

Our orbit fit program uses an all-purpose multidimensional optimization routine known as a simplex method taken from Numerical Recipes ${ }^{10}$. While not always the most efficient method, it is robust enough to use with any data configuration. For a set of short-arc observations, an epoch time is chosen (such as the epoch of the first observation). Different six-dimensional Cartesian vectors are tested by transforming each into Kepler orbits, propagating them to each observation time in the set of observations, and computing the differences between the predicted and observed look-angle vectors. One obvious way to measure this difference is to take the arc cosine of the vector dot product of the two normalized look vectors. However, if the angle between the vectors is small, the dot-product is very close to 1.0 , and can lead to round off problems. Instead, we use the magnitude of the vector difference between the two normal vectors (the actual look vector and the predicted one). This gives an excellent approximation of the angle (in radians) between the two vectors if they are sufficiently close, and it can be transformed into a positional error on the sky in arcseconds. The optimization routine "experiments" with various Cartesian coordinate configurations until the sum of the squares of the positional errors is minimized.

For short arcs, there are in general a variety of different orbits (of varying eccentricity) that give relatively good fits to the data, making it difficult to determine a single optimal orbit with no constraints on the solution. Therefore, the ACO software penalizes solutions by how far their eccentricities differ from zero. This penalty is added to the positional error described above, resulting in an optimized solution equivalent to the best-fit circular orbit to the data set.

The problem with this procedure is that highly eccentricity orbits (such as GTOs) can occasionally "mimic" circular orbits over a short arc. In such cases the computed circular orbit parameters are erroneous. In the case of correlated catalog objects where the orbits are known, it is possible to simply remove higheccentricity orbits from the database. For untracked debris, however, high-eccentricity objects can potentially "pollute" the dataset.

In order to compute statistics, we must make assumptions about which parameters are random, and which are not. For the purposes of this analysis, the position of objects in their orbits (mean anomaly) at any epoch was considered to be random. Now, functional GEO objects tend to be placed in specific longitudes over the rotating Earth. Objects with geosynchronous periods allowed to drift tend to move in specific, nonrandom patterns around the "stable points" in the eastern and western hemisphere. Because the MODEST telescope is stationed in the western hemisphere, objects that remain in the eastern hemisphere are invisible to the sensor.

However, if GEO debris are created in highenergy satellite breakups, then we can expect that the fragments will receive some sort of delta-velocity at the time of their creation. As a result, the fragments will generally have orbital periods that do not match the rotation rate of the Earth. Therefore, in the frame of the rotating Earth (i.e., from the viewpoint of a fixed observer on the Earth) they will tend to drift in longitude over time - eventually working their way around the Earth on a time scale of days or months. Therefore, any "snapshot" in time from any longitude will essentially be a random sample of GEO breakup fragments.

Any given circular orbit (defined by mean motion MM, inclination $\mathrm{i}$, and ascending node $\Omega$ ) may or may not be visible in a given observation frame, depending on the telescope pointing and the position of the telescope on the rotating Earth. MODEST observations have historically pointed at a single Right Ascension and Declination for half or all of a night. However, due to the finite radius of the Earth and its rotation during the night, such observations do not sample a single region of space, but "sweep out" a region on the GEO belt. This means that each frame must be compared to each orbit to determine if detection was possible.

NASA has been experimenting with using a night-to-night correlation routine to identify 
multiple detections of a single object. However, for the purposes of this analysis, we assume that each telescope pointing session is independent of the others. Therefore, if the same object is seen on two different nights, this is assumed to be a random event. Again, this assumption probably breaks down for true geosynchronous objects, but should be approximately true for debris.

The statistical method takes a single circular orbit (MM, i, $\Omega$ ) and tests a full spectrum of starting positions (usually 1,000 test orbits evenly spaced around $360^{\circ}$ in mean anomaly). Each orbit is tested to see if it could have been observed (seen four times within the frame within the appropriate rate box) during the session. The fraction that could have been observed represents the probability that that particular orbit family (all orbits with that MM, $i$, and $\Omega$ ) could have been observed. This is repeated for all sessions of interest and the detection probabilities summed.

Assume for a moment that a particular orbit family had a 0.25 chance of being observed over a set of sessions. Assume also that one object was observed with those orbits parameters in that set of sessions. This means that that one object represents approximately $1 / 0.25 \approx 4$ objects in that particular orbit family (the true answer is \pm some value based on finite statistical sampling). The inverse of the detection probability becomes the "weight" applied to each detection. Using this method, it is possible to build up statistical distributions of orbit families, even if there was not a $100 \%$ chance to see an object in a particular orbit. Note that as the probability of detection exceeds 1.0 , then it becomes increasingly likely that a particular object would be seen multiple times. This is because the probability is actually the timeintegrated average detection rate, and the actual number of detections is a Poisson sample of the time-integrated rate. In the case of probability greater than one, each observation will be weighted by a number less than one. Note, also, that if the detection probability of a particular orbit is zero, then we are unable to make estimates of the population.
By stepping systematically through all orbit possibilities, a map can be constructed that shows the coverage of a particular set of telescope observations. Figure 1 shows just such a map assembled from the 130 nights of MODEST data. Some orbit regimes have been over-sampled, with high likelihood of seeing the same objects 4 or 5 times, while other regions have detection probabilities below 1 . The white vertical stripe around $280^{\circ}$ Right Ascension of the Ascending Node indicates a small subset of orbits could never have been seen in the MODEST telescope over this time because it was simply not pointed in the right direction to observe them.

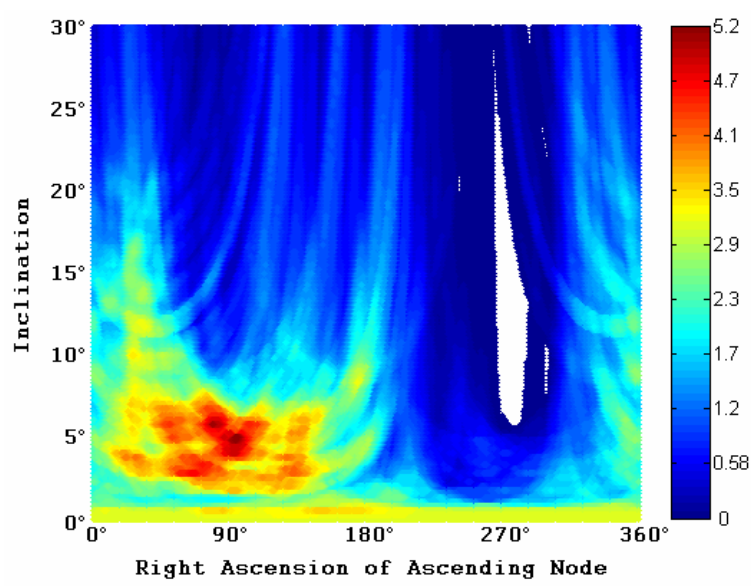

Fig. 1: This color map shows the composite detection probabilities for objects in geosynchronous orbits at different ascending nodes and inclinations based on the MODEST observation sessions from 2002 to 2006 (a total of 130 nights). The probability of detection (or more accurately the time-integrated detection rate) is shown on the scale at the right. As can be seen, many orbit regimes have been overobserved, while at least a small fraction of the environment has not been observed at all (represented by the white vertical region around $280^{\circ}$ Right Ascension of the Ascending Node). This information can be used to remove the observation biases of the objects seen in these particular observation sessions. Despite the irregular coverage, there is sufficient information to arrive at good estimates of the total population of the GEO regime (to the limiting magnitude of the telescope). Note that this kind of information is also useful in scheduling future observations to cover regions not yet seen. 
One thing to notice about this chart is that a simple scatter plot of detected orbits will not tell the story of the actual population in orbit. There will probably be many more objects detected in the high-rate (red) regions than the low-rate (blue) ones, but that does not necessarily translate into more objects in "red" orbits than "blue" orbits. Figures 2 and 3 shows the distribution of objects actually detected by MODEST.

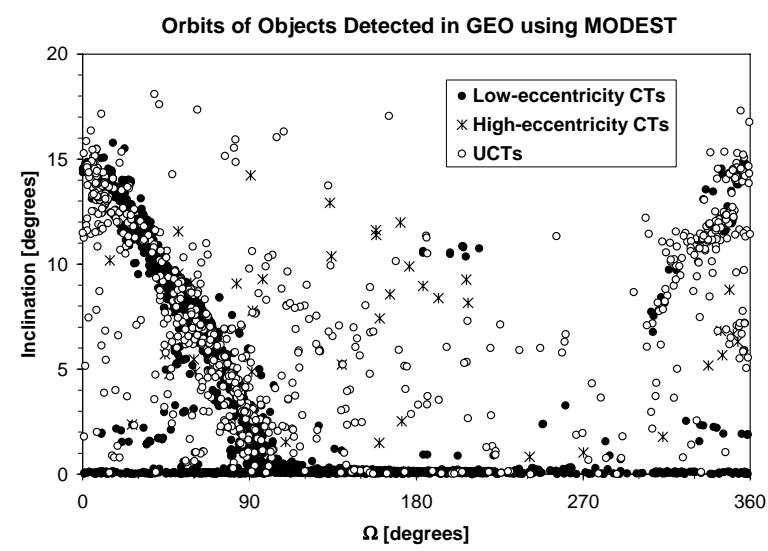

Fig. 2: This figure shows the calculated orbit inclination and right ascension of ascending node $(\Omega)$ of each object detected by MODEST using the Assumed Circular Orbit (ACO) approximation. "CTs" refers to "correlated targets", or objects tracked by the SSN. "UCTs" ("uncorrelated targets") are objects not correlated with any tracked satellite. Typical GEO objects follow a prominent path (the GEO "loop") due to the long-term precession of their orbits. Star symbols show the ACO orbits of CT objects known to have high eccentricities (e $>0.1$ ). These objects do not tend to fall along the GEO "loop". Note that some of the UCT objects are perhaps also in high-eccentricity orbits as well, we simply do not have the capability yet to tell. Many, however, fall on or near the GEO "loop", which indicates a population of untracked objects in GEO-like orbits.

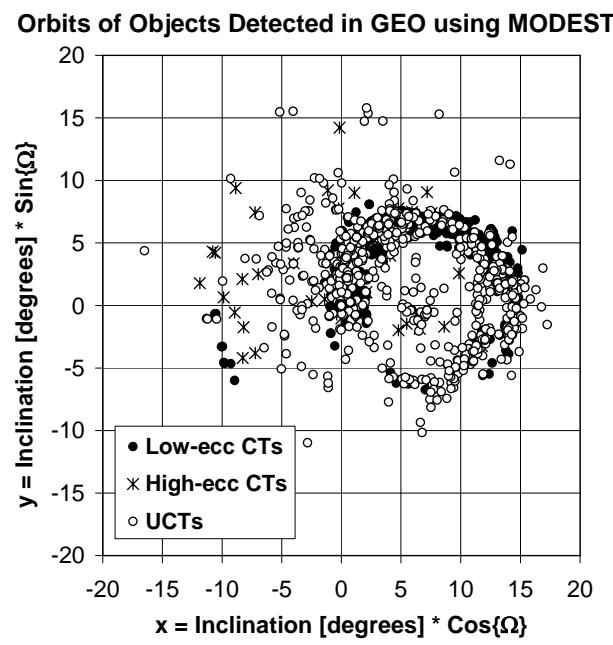

Fig. 3: This figure shows the same data shown in Figure 2, but in a polar coordinate system approximating the pointing of the orbit angular momentum vector $(\mathrm{x}=$ inclination * $\operatorname{Cos}\{\Omega\}, y=$ inclination $* \operatorname{Sin}\{\Omega\})$. The GEO "loop" is clearly visible in this coordinate system as a circle on the right side of the chart with a "radius" of about $7.5^{\circ}$. Again, note that many of the UCTs observed could well be due to highlyelliptical orbits "masquerading" as GEO objects, especially those far from the GEO precessional "loop".

\section{ANALYSIS}

Using the statistical techniques described above, we have assigned to each detected object an ACO orbit, and a weight based on the timeintegrated detection probability. We can now look at the magnitude distribution of all the objects detected. Figure 4 shows this distribution, which should be analogous to a size distribution, although without detailed albedo information, it is difficult to convert directly from absolute magnitude to size correctly.

In LEO, radar observations indicate that the distribution of fragmentation debris with size can be described as a power law, given by ${ }^{11}$ 


$$
C(x) \propto x^{-1.6},
$$

where $C(x)$ is the number of objects larger than a given size $x$. Assuming that the shape and albedo distributions of debris over the size interval of interest are approximately constant, then the intensity of the light from an object should be approximately proportional to the square of the object size $x^{2}$. Therefore, the magnitude $M$ should be

$$
\begin{aligned}
M & =-2.5 \log _{10} x^{2}+\kappa_{1}, \\
& =-5 \log _{10} x+\kappa_{1}
\end{aligned}
$$

where $\kappa_{1}$ is a constant. This means the magnitude distribution of such debris would be

$$
C(m) \propto\left(10^{-\frac{M}{5}}\right)^{-1.6} \propto 10^{0.32 M} .
$$

The differential form of this curve is shown by the dashed line in Figure 4. As can be seen from the figure, the smallest/dimmest objects $(M>$ 15) have a magnitude-dependent slope consistent with the size distribution of fragmentation debris seen in LEO. Therefore, it is reasonable to assume that the population of GEO objects below about $15^{\text {th }}$ magnitude is predominantly composed of fragmentation debris, and those brighter then $15^{\text {th }}$ magnitude are not (perhaps being composed mostly of intact objects).

This analysis opens up the possibility of splitting the population into primarily fragmentation and non-fragmentation components, based only on the absolute magnitude. These populations can then be investigated for their true orbit distributions, based on the statistical weightings. Figure 5 shows these two orbit density populations.

The bright/large object orbit distribution is (mostly) consistent with a distribution of objects that evolved off the GEO ring over many years. The dim/small object distribution shows that some objects have similar orbit distributions to the brighter objects, but there are several "clumps" that indicate families of objects that were created with similar orbit distributions and evolved together. One clump at $\mathrm{x} \sim 11^{\circ}, \mathrm{y} \sim-4^{\circ}$ dominates the small object population, and may represent one or more breakup clouds. This cloud is in a region known to be associated with debris from the 1992 breakup of a Titan 3C-4 Transstage ${ }^{12}$.

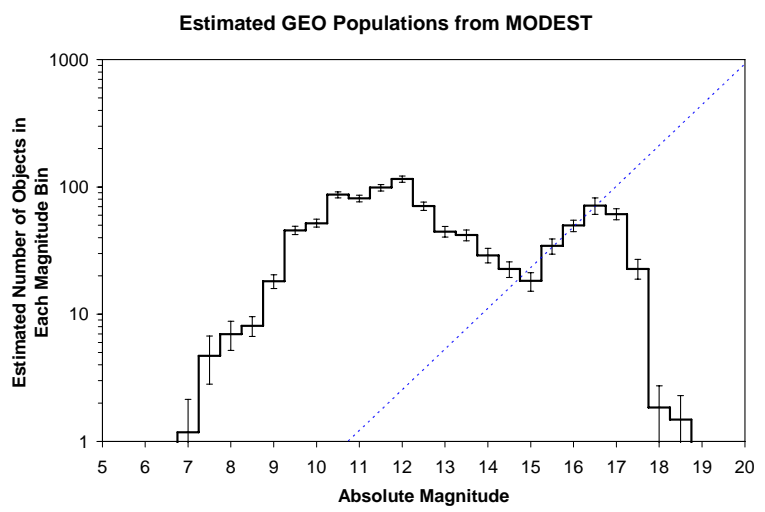

Fig. 4: This figure shows the estimated debiased population of objects in GEO based on MODEST data in 0.5 absolute magnitude (brightness corrected to $36,000 \mathrm{~km}$ distance and $0^{\circ}$ solar phase angle) bins. Larger magnitudes represent dimmer (and probably smaller) objects. The error bars represent the 1 -sigma sampling error of the observations. The drop-off above $\sim 17^{\text {th }}$ magnitude is due to the sensitivity limitations of the MODEST telescope. The distribution is bimodal, similar to what is seen in LEO, where the largest objects are intact spacecraft and rocket bodies, and the smaller objects are primarily fragmentation debris. The line represents the theoretical fragmentation debris curve with a size-dependent slope derived empirically from LEO radar observations (Eq. (3), but on this graph given in differential form). Note that the smaller/dimmer objects seem to have a size/magnitude distribution consistent with the empirical LEO fragmentation debris curve. For reference, $17^{\text {th }}$ magnitude objects are $\sim 30 \mathrm{~cm}$ in diameter and $12^{\text {th }}$ magnitude objects are $\sim 300 \mathrm{~cm}$ in diameter assuming diffuse spheres with albedo 0.2 . 

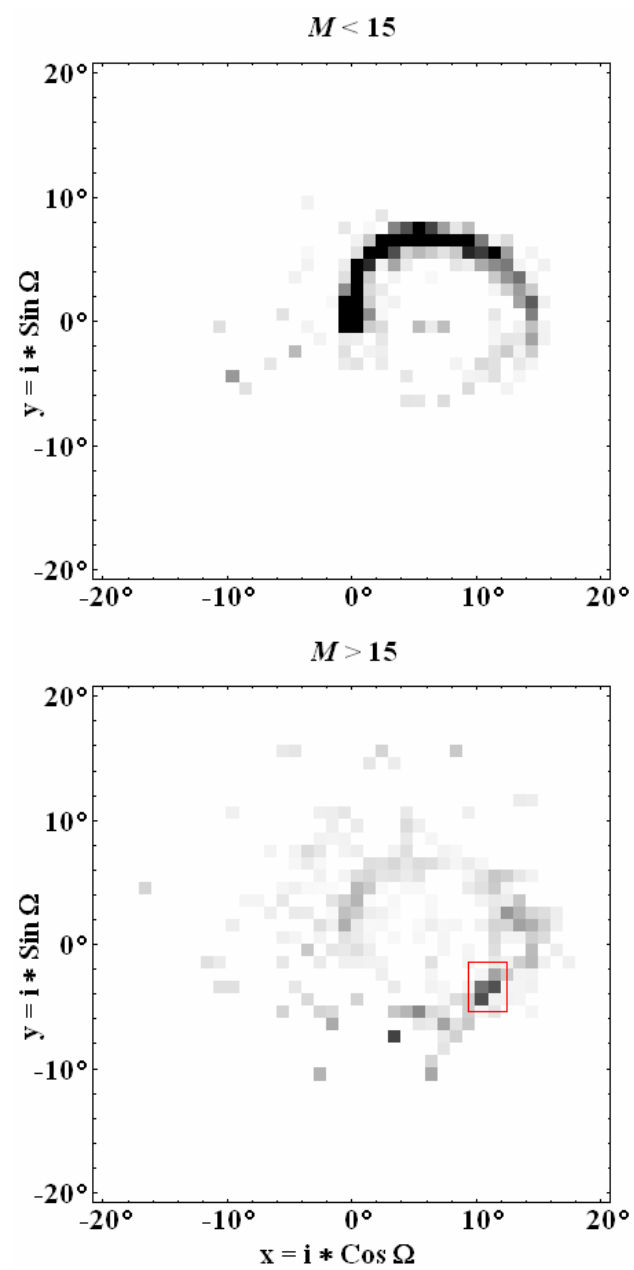

Fig. 5: These two charts show the relative orbit population density in GEO estimated statistically from the MODEST data. The coordinates are equivalent to those shown in Figure 3. The panel on the top is the orbit population density of brighter objects with absolute magnitude $M<15$ - a population probably dominated by intact objects and nonfragmentation debris. The panel on the bottom is the same plot, but for dimmer objects with absolute magnitude $M>15$. These objects appear to be primarily composed of fragmentation debris. The relative darkness of each $1^{\circ} \times 1^{\circ}$ square is proportional to the estimated number of true objects in that bin. Note that the grey levels are consistent between the two charts. The single dark square on the lower chart at $\mathrm{x} \sim 3^{\circ}, \mathrm{y} \sim-8^{\circ}$ is in a region with very poor observational coverage, and that box is represented by a single detected object, so the true population there is highly uncertain. The boxed region at $\mathrm{x} \sim 11^{\circ}, \mathrm{y} \sim-4^{\circ}$ marks a possible breakup cloud discussed in the text and in Figure 6.
Using Figure 5, it is possible to isolate the prominent cloud at $\mathrm{x} \sim 11^{\circ}, \mathrm{y} \sim-4^{\circ}$ and analyze the properties of the objects. For this analysis, all detected objects (not including higheccentricity CTs) with x-values between $9.5^{\circ}$ and $12.5^{\circ}$ and $y$-values between $-5.5^{\circ}$ and $-1.5^{\circ}$ were selected. Figure 6 shows the debiased magnitude distribution of these objects, this time using a cumulative distribution. The size/magnitude distribution is indicates that these objects probably came from one or more breakup events.

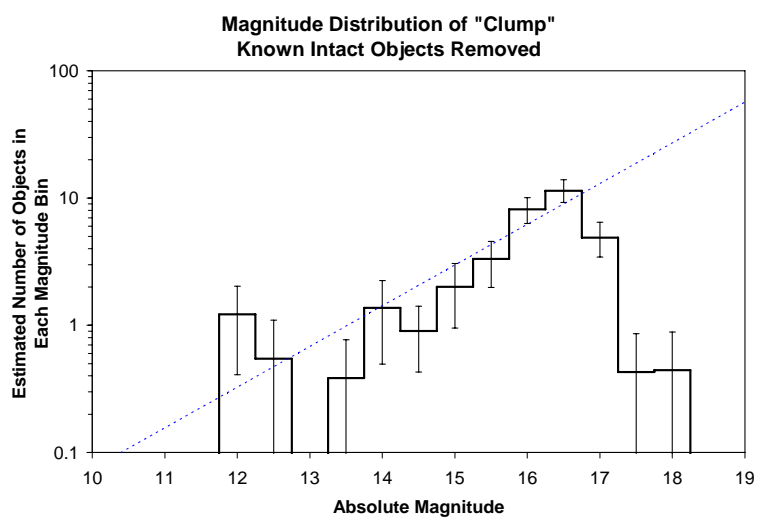

Fig. 6: This is an absolute magnitude distribution of objects in the "clump" region (known intact CTs are removed) located at approximately $\mathrm{x} \sim 11^{\circ}, \mathrm{y}$ $\sim-4^{\circ}$ in the lower panel of Figure 5. The numbers on the $\mathrm{y}$-axis represent the actual statistically-estimated numbers of objects in orbit estimated from the observations. Note that the roll-off above $\sim 17^{\text {th }}$ magnitude is due to the limit of the telescope sensitivity. The dotted line is the converted size distribution given in Eq. (3). The magnitude/size distribution is consistent with a population of fragmentation debris from one or more breakups. For reference, $17^{\text {th }}$ magnitude objects are $\sim 30 \mathrm{~cm}$ in diameter and $15^{\text {th }}$ magnitude objects are $\sim 75 \mathrm{~cm}$ in diameter assuming diffuse spheres with albedo 0.2.

So far in this paper, we have looked at the relationships of inclination, ascending node, and magnitude. The ACO approximation also produces a measure of the mean motion, although the actual value of the mean motion is a function of both the unknown eccentricity and true mean motion of the object. Figure 7 shows the relationship between mean motion and absolute magnitude. The qualitative differences 
we noted between bright and dim objects also extends to the mean motion/eccentricity distributions of the orbits. Objects dimmer than $\sim 15^{\text {th }}$ magnitude appear to be from orbits with significantly different periods than true geosynchronous objects, or have eccentricities different from circular orbits, or some combination of the two. This is consistent with those objects being fragmentation debris created with some delta-velocity. However, this effect may also be due to high area-to-mass objects perturbed by solar radiation pressure. In either case, there are fundamental differences between the brighter and dimmer object populations.

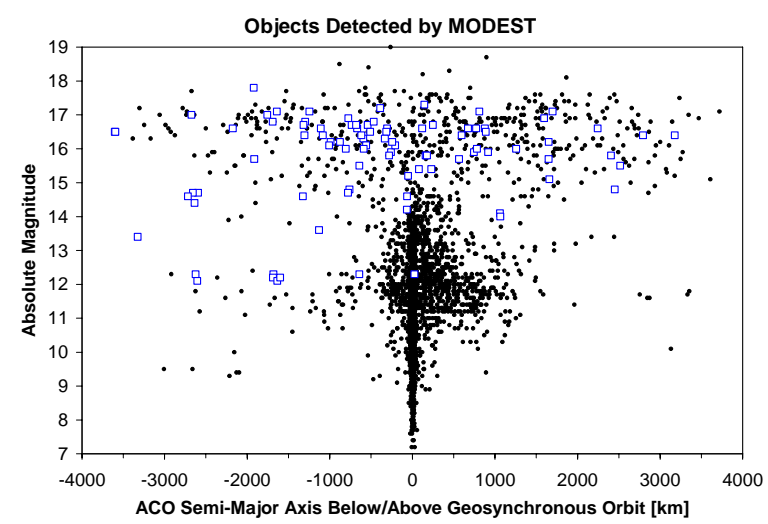

Fig. 7: The scatter plot of ACO semi-major axis (relative to a true geosynchronous orbit) versus absolute magnitude shows than many of the brighter/larger objects have orbits near the GEO ring. The dimmer/smaller objects show a much broader altitude distribution of orbits below and above "true" GEO, which may be due to a variety of different periods, different eccentricities, or both. Note the difference in the character of orbits of objects below and above $\sim 15^{\text {th }}$ absolute magnitude. The squares represent data from the "clump" shown in Figure 5. Note that high-eccentricity CTs are not plotted in this data set.

\section{CONCLUSIONS}

The MODEST telescope has been able to statistically sample most of the GEO environment over the last 5 years. By removing the biases in the observations, we can now estimate the populations of objects actually in orbit in the GEO regime. We have shown that there is an identifiable population of small objects with size distribution characteristics similar to fragmentation debris seen in LEO and orbit characteristics different from the population of intact GEO objects. These small objects show clumps that indicate families of objects - possibly debris from satellite breakups.

This study highlights the need for global surveys of the entire GEO environment and the need for bias removal procedures. In future MODEST campaigns, we will need to plan our observations so that the coverage is more evenly distributed, and that we obtain better coverage over shorter time scales - preferably one year in order to monitor changes in the GEO environment on that time scale.

\section{REFERENCES}

1. N. Johnson, D. Whitlock, P. Anz-Meador, M. E. Cizek, and S. Portman. History of OnOrbit Satellite Fragmentations, $13^{\text {th }}$ Edition, JSC-29517, NASA Johnson Space Center, Houston, TX, 2004.

2. T. Schildknecht, R. Musci, and M. Ploner. Optical Surveys for Space Debris in GEO What About NEOs?, Proceedings of the International Workshop on Collaboration and Coordination Among NEO Observers and Orbital Computers, Kurashiki, Japan, 2001.

3. T. Schildknecht, R. Musci, M. Ploner, W. Flury, J. Kuusela, J. de Leon Cruz, L. de Fatima Dominguez Palmero. Probing the Small-Size Debris Environment in the Geostationary Ring, Proceedings of the 2002 AMOS Technical Conference, Maui, HI, 2002.

4. T. Schildknecht, R. Musci, W. Flury, J. Kuusela, J. de Leon, and L. de Fatima Dominguez Palmero. Optical Observations of Space Debris in High-Altitude Orbits, Proceedings of the Fourth European Conference on Space Debris, ESA SP-587, Darmstadt, Germany, 113 - 118, 2005.

5. M. Matney and E. Stansbery. What are Radar Observations Telling Us About the Low-Earth Orbital Debris Environment?, 
Proceedings of the Third European Conference on Space Debris, ESOC, Darmstadt, Germany, 19-21 March, 2001, pp. $43-48$.

6. K.S. Jarvis, T. L. Parr-Thumm, K. Jorgensen, et al. CCD Debris Telescope Observations of the Geosynchronous Orbital Debris Environment, Observing Year: 1999, JSC-29712, NASA Johnson Space Center, Houston, TX, 2002.

7. M.J. Matney, J. Africano, K. Jarvis, T. Thumm. Extracting GEO Orbit Populations from Optical Surveys, Proceedings of the 2002 AMOS Technical Conference, Maui, HI, 2002.

8. K.F. Gauss. Theoria motus corporum coelestium in sectionibus conicis solem ambientium, Hamburg, F. Perthes and I.H. Besser, 1809.

9. P. Seitzer, K. Jorgensen, J. Africano, T. Parr-Thumm, M. Matney, K. Jarvis, and E. Stansbery. Results from the NASA/Michigan GEO Debris Survey, Proceedings of the AMOS 2004 Technical Conference Proceedings, Maui, HI, 2005.

10. Numerical Recipes in Fortran 77 The Art of Scientific Computing, $2^{\text {nd }}$ Edition, Vol. 1, Cambridge University Press, p 403, 1992.

11. N. Johnson, P. Krisko, J.-C. Liou, and P. Anz-Meador. NASA's New Breakup Model of EVOLVE 4.0, Advances in Space Research, Vol. 28, pp. 1377-1384, 2001.

12. E. Barker, M. Matney, J.-C. Liou, K. Abercromby, H. Rodriguez, M. Horstman, and P. Seitzer. Comparison of Orbital Parameters for GEO Debris Predicted by LEGEND and Observed by MODEST: Can Sources of Orbital Debris be Identified?, Proceedings of the AMOS 2006 Technical Conference Proceedings, Maui, HI, 2006. 